\title{
Right-sided heart reperfusion in pediatric patients with left ventricular assist device
}

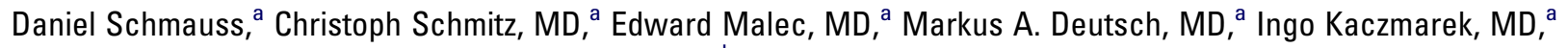 \\ Andre Beiras-Fernandez, MD, ${ }^{a}$ Matthias Loebe, PhD, ${ }^{\mathrm{b}}$ Bruno Reichart, MD, ${ }^{\mathrm{a}}$ and Ralf Sodian, MD, ${ }^{\mathrm{a}}$ Munich, Germany, \\ and Houston, Texas
}

$\mathrm{M}$ echanical circulatory support is important in the bridge to transplantation and bridge to recovery, especially in pediatric patients with end-stage heart failure. Right ventricular failure is a complication and major determinant of survival after implantation of a left ventricular assist device (LVAD). However, an implantable right ventricular assist device (RVAD) is not often clinically used for 3 reasons: (1) It is difficult to predict whether the pediatric patient requiring an LVAD will also require implantation of an RVAD; (2) right ventricular failure recovers more quickly than left ventricular failure; and (3) there is limitation of space in a pediatric patient. ${ }^{1}$ Therefore, we recently developed a safe procedure to preserve right ventricular function during implantation of an LVAD in pediatric patients.

\section{Clinical Summary}

A 4-year-old girl $(16 \mathrm{~kg}$ ) with end-stage cardiomyopathy was transferred to the pediatric intensive care unit. The patient's condition deteriorated rapidly, with lung edema, tachycardia, and signs of multiorgan failure necessitating inotropic support and ventilation. Because of the patient's ongoing tachyarrhythmia, we decided to implant a mechanical circulatory assist device. Echocardiography revealed left and right ventricular dysfunction, but it was not possible to predict before implantation whether the patient would require an LVAD or a biventricular assist device. Thus, we transferred the patient to the operating room with the plan to implant an LVAD with the option to implant a biventricular assist device if the patient also required an RVAD.

At the beginning of the LVAD (Berlin Heart; Berlin Heart Inc, Berlin, Germany) implantation, cannulation for cardiopulmonary bypass $(\mathrm{CPB})$ is performed in the usual fashion. After completion of the LVAD implant procedure, the device supports the left ventricle and CPB can be stopped. In this situation, the ability of the right

From the Department of Cardiac Surgery, ${ }^{a}$ Ludwig-Maximilian University, Munich, Germany; and Michael E. DeBakey Department of Surgery, Baylor College of Medicine, Houston, Texas.

Received for publication Sept 25, 2007; accepted for publication Oct 2, 2007.

Address for reprints: Ralf Sodian, MD, Department of Cardiac Surgery, Ludwig-Maximilians-University, Marchioninistr. 15, 81377 Munich, Germany (E-mail: ralf.sodian@med.uni-muenchen.de).

J Thorac Cardiovasc Surg 2008;135:953

$0022-5223 / \$ 34.00$

Copyright (c) 2008 by The American Association for Thoracic Surgery doi:10.1016/j.jtcvs.2007.10.073 ventricle to provide sufficient flow through the pulmonary circuit to fill the device may be a problem and weaning from CPB is not possible. Therefore, a $12 \mathrm{~F}$ aortic cannula is placed into the pulmonary trunk, the aortic cannula is clamped, and the arterial line is connected to the pulmonary cannula. Afterward, right-sided heart reperfusion is initiated. During reperfusion, the LVAD function is adjusted, and medical therapy to support right ventricular function is initiated. After a short period of right-sided heart reperfusion, the filling of the LVAD improves significantly and weaning from right heart $\mathrm{CPB}$ can be performed. Our patient did not require a right ventricular or biventricular support system and underwent transplantation successfully after 3 weeks of LVAD support.

\section{Discussion}

Right ventricular failure is a crucial problem after LVAD implantation because of insufficient filling of the left ventricle resulting from the inability to wean from $\mathrm{CPB}$ and because right ventricular failure often is refractory to catecholamines and nitric oxide. In this situation the implantation of an RVAD is often necessary. ${ }^{1}$

There is limited space for cannulas and devices in pediatric patients, and therefore implantation of an RVAD may be complicated. Loebe and colleagues ${ }^{2}$ previously reported a technique for preserving right ventricular function during implantation of an LVAD in adult patients. They cannulated the pulmonary trunk at the beginning of the operation, using this as a vent during the LVAD implantation and as support of pulmonary perfusion after the LVAD implantation. ${ }^{2}$ This procedure is helpful in adult patients but difficult to perform in newborns and smaller children because of numerous tubes, connectors, and clamps.

With the use of our technique, excellent right ventricular support after LVAD implantation was provided. The filling of the LVAD improved, and weaning from CPB was performed under stable conditions without stressing the right ventricle. Right ventricular failure recovered rapidly, and there was no need for additional mechanical right ventricular support. The postoperative course was uneventful, and the patient received a donor heart after 20 days of support.

This technique, in combination with medical therapy, provides good right ventricular support and avoids implantation of an RVAD. It is simple to implement and has proved to be effective, especially in pediatric patients.

\section{References}

1. Furuka K, Motomura T, Nosé Y. Right ventricular failure after left ventricular assist device implantation: the need for an implantable right ventricular assist device. Artif Organs. 2005;29:369-77. Review.

2. Loebe M, Potapov E, Sodian R, Kopitz M, Noon GP. A safe and simple method of preserving right ventricular function during implantation of a left ventricular assist device. J Thorac Cardiovasc Surg. 2001;122: 1043 